in ihrer Antwort auf einen eLetter zu ihrer Publikation auf der Homepage der Zeitschrift „Fertility and Sterility“ feststellen.

Die Auswertung der Metaanalyse lässt nach Ansicht der Autoren den Schluss zu, dass die Kombination von einmal verabreichtem, gut verträglichem Corifollitropin mit einer fixen Dosis eines GnRHAntagonisten ab Tag 5 der Stimulationsbehandlung eine ähnliche Schwangerschaftsrate ermöglicht wie die tägliche Injektion von rekombinantem follikelstimulierenden Hormon (FSH). Darüber hinaus werden signifikant mehr Oozyten allgemein, Metaphase-II-Oozyten sowie
Embryonen erzielt bei gleichzeitig geringerer FSH-Dosis. In der Kombinationsgruppe benötigten die Ärzte nur zwei zusätzliche Tage, um eine Reifung der Oozyten zu erreichen.

Peter Leiner

Youssef MAM et al. Is there a place for corifollitropin alfa in IVF/ICSI cycles? A systematic review and meta-analysis. Fertility and Sterility 2012; 94/4: 876-85

Kommentar: Das inzwischen in Deutschland eingeführte Corifollitropin alfa wurde bisher hauptsächlich bei „low-respondern“ eingesetzt. Hier führt es bei eingeschränkten
Anti-Müller-Hormon (AMH)-Werten aufgrund seiner hohen primären Anflutung zu einem verbesserten Follikelwachstum verglichen mit der täglichen Injektion. Bei „normal-respondern" (normale AMH-Werte) ist es fraglich, ob die Einmaldosis einen Vorteil bringt gegenüber einer individuell angepassten, täglichen Injektion. Beim polyzystischen Ovarialsyndrom (PCO) verbietet sich bisher die Injektion aufgrund der überschießenden Wachstumskinetik.

Insgesamt stellt aber die Einführung von Corifollitropin alfa im Spektrum der Therapiemöglichkeiten bei ART eine gute Ergänzung dar.

Prof. Dr. Thomas Katzorke

\title{
Ovarialkarzinom: präoperatives CA125 als Prognosemarker
}

\section{Bei den malignen epithelialen Ovarialtumoren lassen sich diejenigen mit niedrigem Malignitätspotenzial abgrenzen. Bei der Beurteilung des Therapieerfolges im Sinne einer 5-Jahres-Rezidivfreiheit und 5-Jahres- Gesamtüberlebenszeit erwiesen sich erhöhte CA125-Spiegel als unabhängiger prognostischer Biomarker.}

O varialtumore mit niedrigem Malignitätspotenzial (borderline Ovarialtumor) machen etwa $10-15 \%$ aller epithelialen ovarialen Neoplasien aus. Betroffen sind überwiegend jüngere Frauen, sodass der Erhaltung der Fertilität von hoher Priorität ist. In den meisten Fällen wird die Diagnose im Stadium I gestellt und die Heilungsrate nach der Operation beträgt bis zu 95\% über einen Nachverfolgungszeitraum von zehn Jahren. Bisher liegen nur wenige Untersuchungen zur Identifikation von Hochrisikogruppen vor. $\mathrm{Zu}$ den prognostischen Faktoren gehören u.a. ein fortgeschrittenes Stadium sowie eine konservative Operation, d.h. ovarielle Zystektomie anstelle einer Oophorektomie. Der Tumormarker CA125 gilt als der einzige Serumtumormarker, der in Kombination mit Ultraschall und/oder anderen Biomarkern eine Triage von Patienten mit pelvischen Tumoren sowie das Management bei einer bekannten Anamnese von epithelialen Oviralkarzinomen erlaubt. Die Messung von CA125 erfolgt auch zur Abschätzung des Erfolges einer Chemotherapie bei fortgeschrittenem Ovarialkarzinom. Nun wird vermutet, dass ein erhöhtes präoperatives CA125 ein prognostischer
Marker zur Abschätzung der Überlebensrate im Stadium I beim epithelialen Ovarialkarzinom ist. Auch wurde gezeigt, dass eine Normalisierung von CA125 bei Frühstufen eines Ovarialkarzinoms nach erfolgtem ersten Chemotherapiezyklus mit einer signifikanten Verbesserung der Rezidivfreiheit und der Überlebensrate einhergeht. Bei Ovarialtumoren mit niedrigem Malignitätspotenzial ist die Bedeutung von erhöhten CA125-Spiegeln hingegen noch unklar. Nur bei 56\% der Patienten mit Ovarialtumoren mit niedrigem Malignitätspotenzial lässt sich eine Erhöhung von CA125 nachweisen.

In dieser multinationalen Studie wurde jetzt die prognostische Bedeutung von CA125-Werten $\geq 50 \mathrm{U} / \mathrm{ml}$ bei 940 Patienten mit Ovarialtumoren mit niedrigem Malignitätspotential, die im Zeitraum von 1985 bis 2008 diagnostiziert wurden, untersucht. Die Behandlung erfolgte entweder mit bilateraler Salpingooophorektomie mit/ohne Hysterektomie oder konservativ, fertilitätserhaltend. Ein Multivarianten-Cox-Modell wurde für die Bestimmung von unabhängigen Risikofaktoren für eine Rezidivfreiheit und der Überlebensrate durchgeführt. Ausgehend von der ROC-Kurve wurde ein CA125Wert von $\geq 50 \mathrm{U} / \mathrm{ml}$ als erhöht angesehen. Es zeigte sich, dass CA125 öfter bei serösen als bei muzinösen Tumoren sowie bei fortgeschrittenen FIGO-Stadien (Stadium 2 bis 4 ) als im Stadium 1 erhöht war. Die Rezidivfreiheit betrug nach fünf Jahren $89 \%$ bei erhöhten CA125- und $95 \%$ bei normalen CA125-Spiegeln $(\mathrm{p}<0,05)$. Ähnliches fand sich auch bei der Gesamtüberlebenszeit. Hier betrug die 5-JahresÜberlebenszeit 90\% bei erhöhten CA125und $95 \%$ bei normalen CA125-Spiegeln $(\mathrm{p}<0,05)$. Sowohl für die 5 -Jahres-Rezidivfreiheit wie auch die 5-Jahres-Überlebenszeit erwiesen sich erhöhte CA125Spiegel als unabhängiger prognostischer Faktor. Die Analyse von Untergruppen ergab, dass CA125 nur für seröse Ovarialtumore mit niedrigem Malignitätspotential prognostisch relevant war.

Fazit: Die Studie belegt, dass präoperative erhöhte CA125-Spiegel bei serösen Ovarialtumore mit niedrigem Malignitätspotenzial als prognostischer Biomarker gewertet werden können. Dies erlaubt eine Abschätzung für die Rezidivfreiheit und Gesamtüberlebenszeit. Da die CA125Bestimmung überall verfügbar ist, hilft der Parameter bei der prognostischen Beurteilung eines möglichen Therapieversagens.

Prof. Tino F. Schwarz

Tang A et al.: Prognostic value of elevated preoperative serum CA125 in ovarian tumors of low malignant potential: a multinational collaborative study (ANZGOGO801). Gynecol. Oncol 2012; doi:10.1016/j.gyno.2012.02.031 